\title{
Fluid-structure interaction enhances the aerodynamic performance of flapping wings: a computational study
}

\author{
Toshiyuki NAKATA*, Ryusuke NODA*,** and Hao LIU* \\ *Graduate School of Engineering, Chiba University \\ 1-33, Yayoi-cho, Inage-ku, Chiba 263-8522, Japan \\ E-mail: hliu@faculty.chiba-u.jp \\ **Department of Mechanical Engineering, Kanto Gakuin University \\ 1-5-10 Mutsuura-Higashi, Kanazawa-ku, Yokohama-shi, Kangawa 236-8501, Japan
}

Received: 5 December 2017; Revised: 29 January 2018; Accepted: 5 February 2018

\begin{abstract}
Insect wings change its shape passively by the aerodynamic and inertial forces when flapping, which can greatly affect its aerodynamic performances. In order to confirm the importance of the fluid-structure interaction in flapping wing aerodynamics, we performed computational fluid-structure interaction analyses of a hovering hawkmoth with 'virtual' vacuum conditions that can adjust the effect of the aerodynamic force on the deformation of flapping wings. It is turned out that the large part of the wing deformation, such as the wing twist, is induced by the inertial force as reported previously, but the adjustment of the wing deformation by the aerodynamic force can greatly affect the kinematics and the aerodynamics of flapping wings. While the wing deformation, regardless of the contribution of the aerodynamic force, can increase the aerodynamic power, force and efficiency of flapping wings, the wing deformation adjusted in response to the unsteady aerodynamics of flapping wings can further enhance the aerodynamic performance. These results not only reveal the influence of the wing deformation on the aerodynamic performance of flapping wings, but also point out the great importance of the fluid-structure interaction in the aerodynamics of insect flight and the design of bio-inspired micro aerial vehicles.
\end{abstract}

Keywords : Flapping wing, Flexible wing, Wing deformation, Fluid-structure interaction

\section{Introduction}

Insect flight has been attracting wide attentions from engineers because of its great flight performances and potentials to inspire novel unmanned aerial vehicles (Liu et al, 2016). Insects flap their wings to generate and modulate the aerodynamic forces to manoeuver in three-dimensional space, utilizing a variety of unsteady aerodynamic mechanisms such as the leading-edge vortex (Ellington et al, 1996; Liu et al, 1998), trailing-edge vortex (Bomphrey et al, 2017), rotational circulation and drag (Bomphrey et al, 2017; Dickinson et al, 1999), and clap and fling (Srygley \& Thomas, 2002; Weis-Fogh, 1973).

Unlike manmade aircraft, such as multi-copter type drone, the flapping wings of insects are known to be flexible (Wootton, 1981). The passive shape changes due to the flexibility can have a great impact on the aerodynamic performances of flapping wings. A numerical study (Young et al, 2009) with realistic wing shapes based on the high-speed photogrammetry (Walker et al, 2009) suggested that the twist and camber can enhance the aerodynamic efficiency of the forward flight of locusts. Similar benefits of the wing deformation have been confirmed in the flight of butterflies or beetles in spite of the great differences in flight morphology (Le et al, 2013; Zheng et al, 2013).

The wing is passively deformed by the fluid-structure interaction, because insect wings are flexible and lack any internal musculature. The enhancement of the efficiency is, therefore, achieved by the well-designed multi-scale structure of the insect wings (Wootton, 1992). Numerical analyses of the fluid-structure interaction of flapping wings in 
air (Nakata \& Liu, 2012b) have suggested that the passive wing deformation delays the burst of the leading-edge vortex, adjusting the phase of rotation, so that the flexible wings can generate higher aerodynamic forces efficiently in comparison with the rigid and flat wings.

Because of the flapping, the wing deformation is induced by two kinds of different forces: inertial forces due to the acceleration and deceleration of the wings, and the aerodynamic forces. Both are unsteady, and change the direction with respect to the wing during one wingbeat. The inertial forces are highest at the stroke reversal when the wing acceleration is maximized, while the aerodynamic forces are highest when the wing velocity is higher at around the middle of the wing stroke. The balance of inertial and aerodynamic forces may, therefore, affect the phase of wing deformation and hence the aerodynamic performances. Combes and Daniel (Combes \& Daniel, 2003) suggested that the majority of the deformation of a hawkmoth wing is due to the inertial force, and therefore the wing can be designed relatively easily without considering the fluid-structure interaction. Yin and Luo (Yin \& Luo, 2010) performed numerical analyses of two-dimensional flexible wings with adjusted ratio of inertial and aerodynamic forces, demonstrating that the wing deformation dominated by either of inertial or fluid dynamic forces can enhance the lift forces, but the wing deformation dominated by the fluid dynamic force can give higher efficiency. Sridhar and Kang (Sridhar \& Kang, 2015) also performed two-dimensional fluid-structure interaction analyses at fruitfly scale and water tunnel scale, and reported the strong influence of the fluid density on the structural response of the flexible wings.

In this study, in order to give a further understanding of the kinematic and the aerodynamic effect of the three-dimensional wing deformation induced by fluid-structure interactions, we performed computational analyses of a hovering hawkmoth with flexible flapping wings in a 'virtual' vacuum chamber that can systematically adjust the contribution of aerodynamic force to the deformation of the flapping wings. Based on the analyses, we investigated the effect of fluid-structure interaction on the wing deformation and the aerodynamic performances. Our results clarify the mechanism with which to enhance the aerodynamic performance through the passive wing deformation, pointing out the importance of the fluid-structure interaction in the flapping wing aerodynamics.

\section{Methods}

\subsection{Integrated model of a hovering hawkmoth}

While the wing deformation due to fluid-structure interaction is widely recognized in various insects, a hovering hawkmoth is chosen as a model insect in this study, because its size and capability of hovering are thought to be suitable for the design of bio-inspired micro aerial vehicle. Furthermore, the morphological and kinematic data of

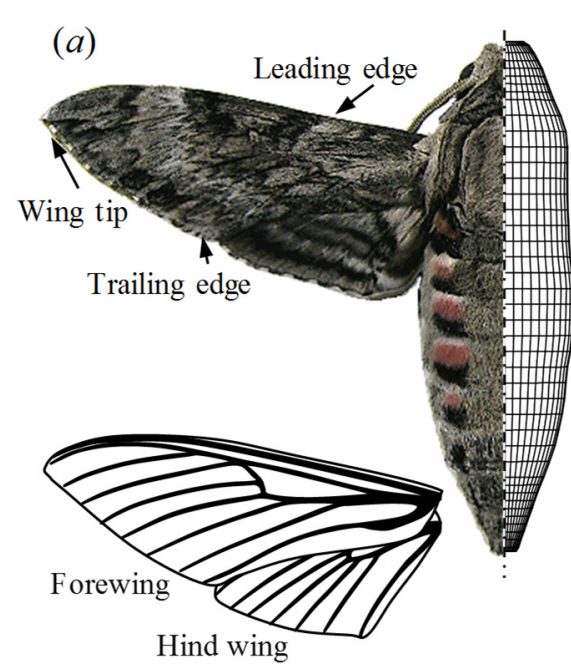

Hind wing
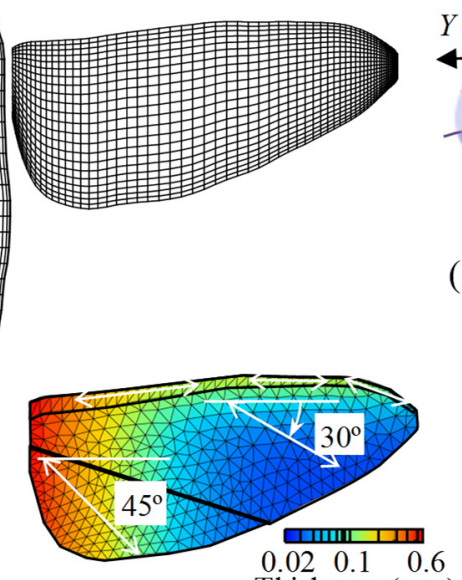
Thickness (mm)
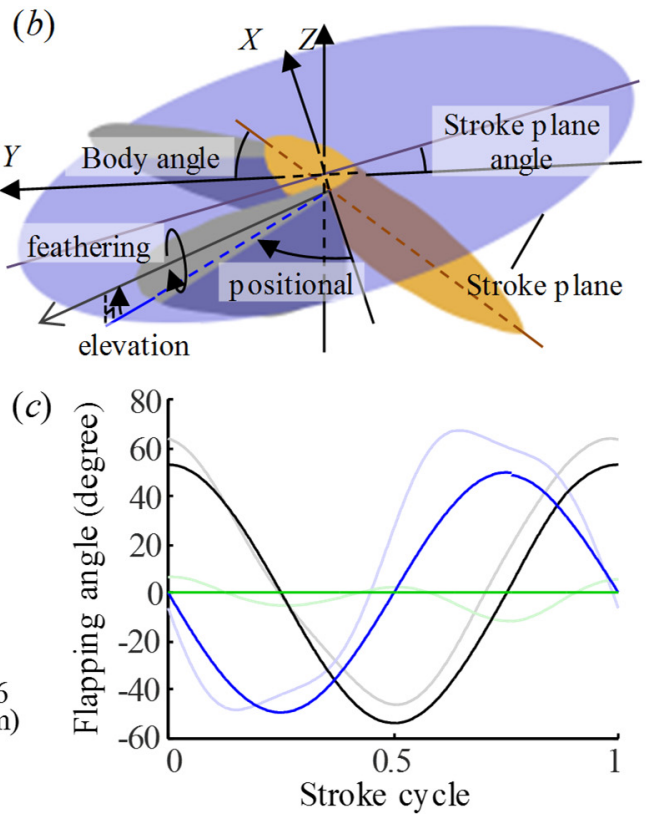

Figure 1 Morphological, kinematic and structural models of a hovering hawkmoth. (a) Agrius convolvuli with the wing venation, and its morphological and structural models for computational fluid-structure interaction analyses. White arrows on the structural model represents the fiber directions to model the anisotropy in the wing of a hawkmoth. (b) Definition of the flapping angles. (c) Time-series of the simplified positional (black), feathering (blue) and elevation (green) angles. The measured kinematics are plotted with thinner lines. 
hovering hawkmoths have been collected very well. In order to integrate the morphological, kinematic, structural and aerodynamic model of a hovering hawkmoth, we utilized a bio-inspired dynamic flight simulator (Liu, 2009; Nakata \& Liu, 2012a). The fore- and hind-wings are modeled as a single unit, and constructed by tracing the outline of the digital image. While the wing area of actual hawkmoths varies during flapping because of the separated fore-and hind-wings, such variation in the wing area is neglected in the current model. The body, left and right wings are modelled separately by the multi-block overset grid method as shown in figure $1 a$. The flapping angles, defined as figure $1 b$, is based on a measurement of a hovering hawkmoth (Willmott \& Ellington, 1997), but is simplified from the original kinematics because the measured kinematics contains the wing deformation (figure 1c). The body angle and the stroke plane angle are 40 and 15 degrees, respectively. The wing deformation is simulated by finite element method that combines Allman triangular and discrete Kirchhoff triangular element. The nonlinear dynamic response of the structure is modelled by introducing an updated Lagrangian formulation. The wing is assumed to have an anisotropy with taper toward the wing tip and the trailing edge (figure 1a). While the actual insect wing has an inherent camber and twist, and asymmetry against the dorsal and ventral bending, the current wing model is assumed to be flat and symmetric. The simplification is necessary because of the lack of the data of detailed wing structure, but the fluid-structure interaction of the flexible flapping wings, and its aerodynamic consequences, are thought to be captured reasonably well by the current model with the anisotropy and the distribution of the wing structure. The details of the structural model can be found in the previous study (Nakata \& Liu, 2012a). In order to model the flapping wing aerodynamics, we utilized a fortified Navier-Stokes solver for the dynamically moving multi-blocked overset grid system. The governing equation for the fluid dynamic model is the unsteady, three-dimensional and incompressible Naiver-Stokes equations. The grids and time steps are verified in the previous studies (Liu, 2009; Nakata \& Liu, 2012a). The parameters used in this study are summarized in Table 1. The weight of the hawkmoth is $1,579 \mathrm{mg}(15.5 \mathrm{mN})$ (Willmott \& Ellington, 1997), and, with the measured wing kinematics and the rigid wing assumption, the simulator could successfully generate the vertical aerodynamic force to support the weight (Liu, 2009). Because of the simplification of the wing kinematics and the wing deformation, the model in this study may not always generate enough amount of aerodynamic forces to hover, but the flight mode is called hovering in this study since the flyer is tethered in the global frame.

\section{2 'Virtual' vacuum conditions}

The structural and fluid dynamic models are combined by a loose coupling method to simulate the dynamic fluid-structure interaction of flexible flapping wings, as summarized in figure 2. In the conventional serial staggered algorithm (Piperno \& Farhat, 2001) utilized in this study, the predictor for the structural displacement at next time step is constructed for the fluid dynamic simulator on the basis of the instantaneous displacement, velocity and acceleration so that the inner iteration can be avoided. In a previous study (Combes \& Daniel, 2003), the vacuum chamber with helium was utilized to confirm the importance of the inertial forces on the flapping wing deformation. In order to achieve a 'virtual' chamber in the simulator, we multiply the density ratio, $r_{v}$, ranging from 0.1 to 1.0 , to the aerodynamic forces that is transferred to the structural dynamic simulator. If the density ratio is reduced down close to zero, the structural deformation is fully determined by the inertial forces. Since the aerodynamics is simulated by a

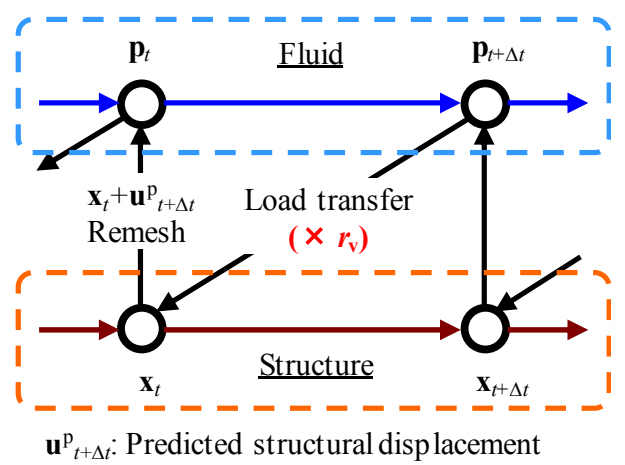

Figure 2 Conventional serial staggered algorithm with the density ratio for 'virtual' chamber. Each cycle goes as follows: (1) Predicting the structural displacement at next time step, $\mathbf{u}^{\mathrm{p}_{t+\Delta t}}$, from the displacement and velocity at current time step using second-order time-accurate predictor. (2) Updating the fluid grid by using the predicted displacement and advancing the fluid system, $\mathbf{p}_{t}$, to next time step, $\mathbf{p}_{t+\Delta t}$. (3) Transferring a fluid pressure to the structure (load transfer). The density ratio, $r_{\mathrm{v}}$, is multiplied to the aerodynamic forces to adjust the aerodynamic effect. (4) Advancing the structure and update the structural state, $\mathbf{x}_{t}$ to $\mathbf{x}_{t+\Delta t}$. 

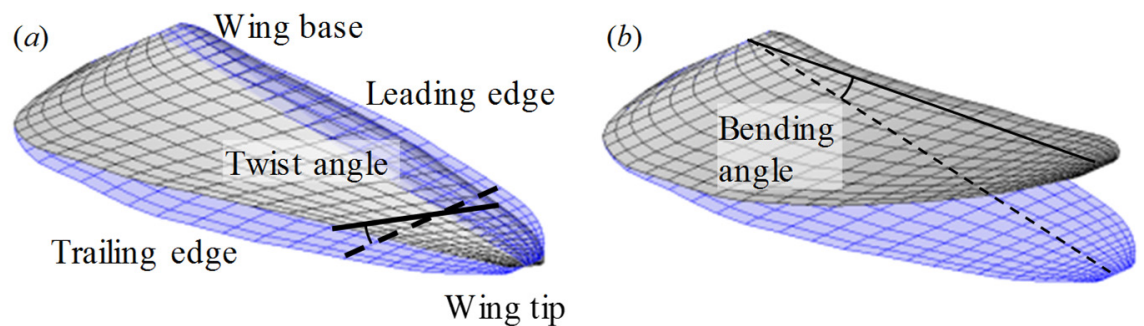

Figure 3 Definitions of $(a)$ the wing twist, and $(b)$ the spanwise bending.

constant density of air regardless of the density ratio, the effect of the changes in the wing deformation due to the 'vacuum' on the aerodynamic performance can be investigated.

\subsection{Kinematic and aerodynamic effect of fluid-structure interaction}

The effect of the fluid-structure interaction on the aerodynamics of flexible flapping wings is evaluated in terms of the wing deformation and the aerodynamic performances. The wing deformation is quantified by the wing twist and the spanwise bending. The twist is defined as the rotation of each cross section about the spanwise axis. For flapping wing aerodynamics, the distal part of the wing is more important, and, therefore, the twist at $80 \%$ of the wing length from the wing base is utilized as a representative (figure $3 a$ ). Note that the spanwise position of the mean chord length is at about $70 \%$ of the wing length from wing base. The spanwise bending angle is defined as the elevation normal to the rigid and flat wing surface at wing tip (figure $3 b$ ). Aerodynamic performance of flapping wings is evaluated in terms of the cycle-averaged vertical force and aerodynamic power consumption to flap the wing, and the aerodynamic efficiency. The aerodynamic efficiency is the ratio between the induced power, which is computed by the product of the vertical force and the mean downwash velocity on the virtual surface in the vicinity of the wing (Nakata \& Liu, 2012a), and the aerodynamic power consumption. The efficiency, therefore, denotes how much power is used to generate the vertical force to achieve hovering out of the input power for moving the wing in air.

\section{Results}

The numerical model revealed that the twist and the bending are affected by the aerodynamic forces. Figure 4 illustrates the time-series and the amplitude of the nose-down twist and the upward bending that are clearly altered in the magnitude and the phase by the reduction of aerodynamic forces, while at most 70.5 and $53 \%$ of the twist and the bending in the air are due to the inertial forces if they are estimated from the amplitude as shown in figure 4bi,bii. Note that the density ratio of 0.1 still contains the $10 \%$ of aerodynamic forces in the air, and the contribution of the inertial forces can be slightly lower than the values shown here. The twist and the bending are maximized at the stroke reversal when the inertial forces are maximized (figure 4ai,aii). With the existence of the air, the maxima is increased, and is delayed from the stroke reversal, which is thought to be due to the aerodynamic damping effect. There are clear second peaks in both twist and bending before the end of each half-stroke with higher density ratio.

The wing deformation affects the wing kinematics at the distal part of the flapping wings, as shown in figure 5 . The feathering angle is slightly advanced by the wing deformation (figure 5ai), and its velocity is greatly increased at the stroke reversal especially with higher density ratio (figure 5 aii). The bending increases the wingbeat amplitude and skews the profile of the wing tip velocity so as to advance the timing of maximum wing tip velocity, which is clearer when the aerodynamic contribution is higher (figure $5 b i$, bii).

The kinematic changes have a great impact on the aerodynamic performance. Figure 6 illustrates the time-series of the aerodynamic powers and the vertical forces generated by the rigid and flexible wings with various density ratios. With the rigid wings, the aerodynamic powers and forces are maximized slightly after the middle of the half-stroke when the angular velocity is highest, because several aerodynamic mechanisms, such as the force due to added mass and the rotational drag that is due to the pitch-up of the wing for stroke reversal, are combined in addition to the translational lift force. The contribution of the aerodynamic forces to the wing deformation affects the aerodynamic powers and forces similarly; the wing deformation enhances the powers and the forces at the beginning of each half-stroke, reduces the maxima, delays the timing of the peak, and reduces the rate of the reduction of the powers and forces before the stroke reversal. These changes are much clearer with the higher density ratio, and, therefore, are thought to be due to the result of the fluid-structure interaction. 
As shown in figure 7, the cycle-averaged aerodynamic powers and vertical forces, and the aerodynamic efficiencies
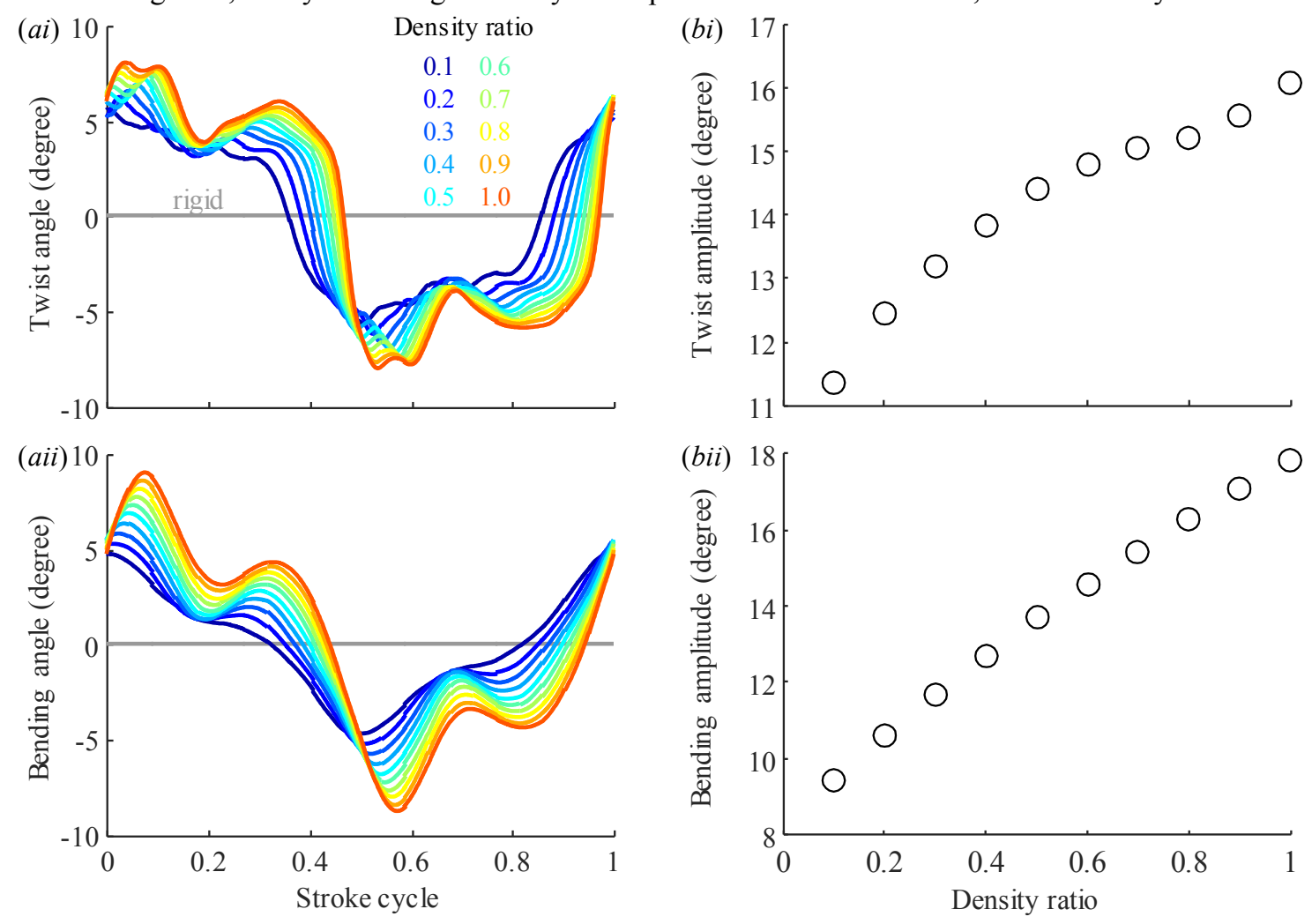

Figure 4 Effect of the density ratio on $(a)$ the time-series and $(b)$ the amplitudes of $(i)$ the wing twist and (ii) the spanwise bending.

(ai)
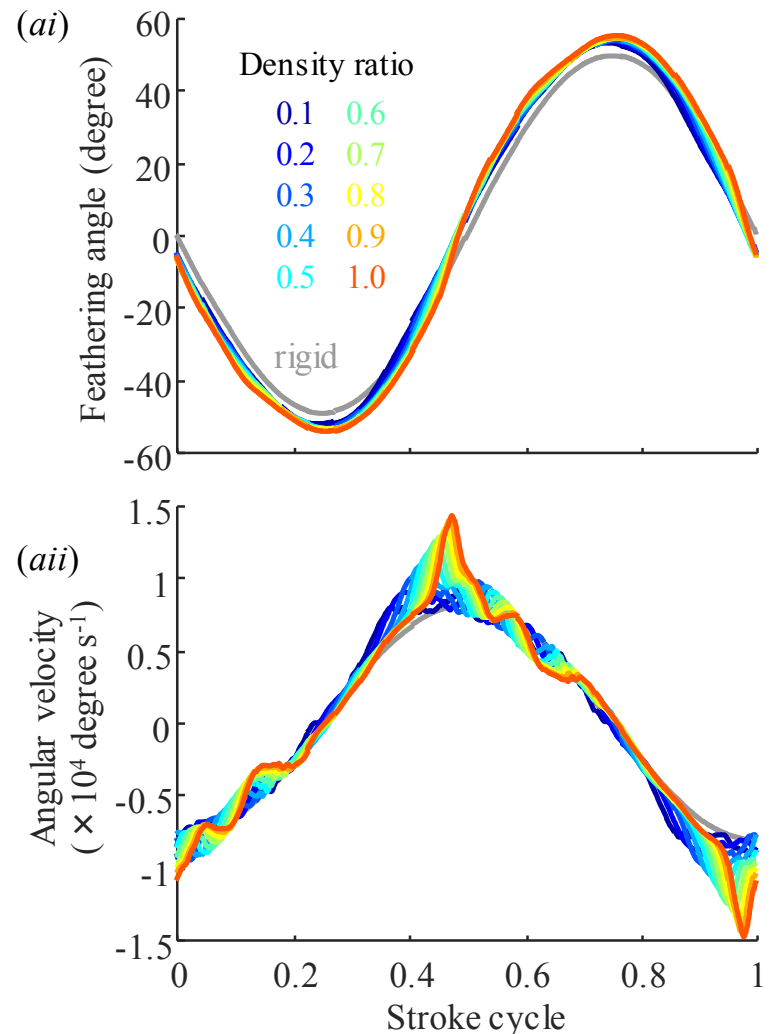

(bi)
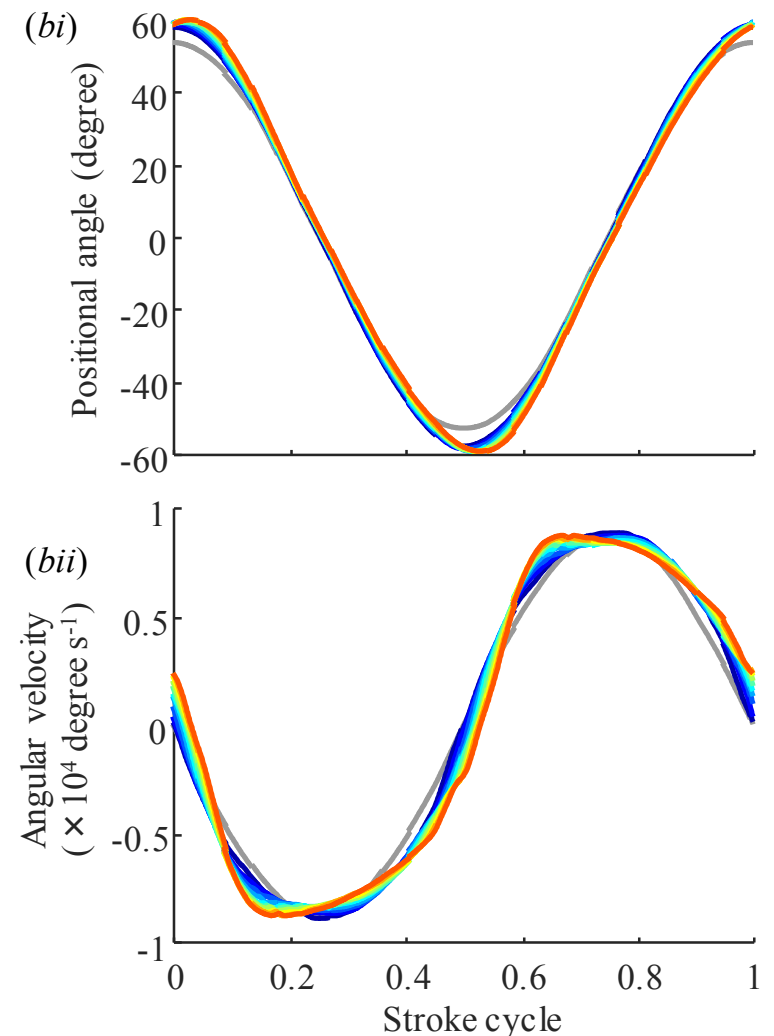

Figure 5 Effect of the density ratio on the time-series of the (ai) feathering and (bi) positional angles and (ii) the angular velocities. 


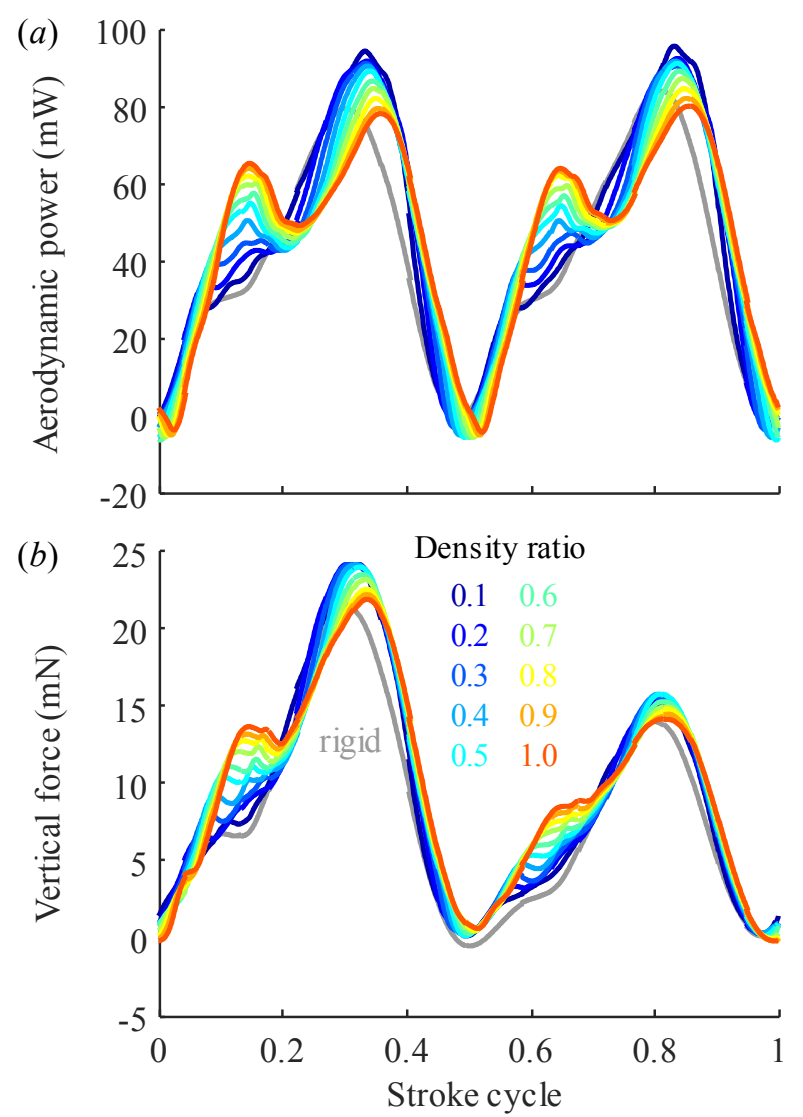

Figure 6 Time-series of $(a)$ the aerodynamic powers and $(b)$ the vertical forces of the rigid (grey) and flexible flapping wings with various density ratios.

are increased by the wing deformation at whole range of the density ratio in comparison with the rigid wing. The aerodynamic force is monotonically increased with increasing density ratio, while the aerodynamic power is increased up to the density ratio of 0.6 , but is not increased further with higher density ratio. As a result, the aerodynamic efficiency is monotonically increased with increasing density ratio, but the increase is much clearer with higher density ratio than 0.6.

\section{Discussion}

In this study, it is clearly shown that the wing deformation of the flexible flapping wings of insects is under the effect of the aerodynamic force, while the inertial force dominates the wing deformation as suggested in a previous study (Combes \& Daniel, 2003). In terms of the amplitude of the wing deformation, large part of the wing twist in air, which is suggested to enhance the aerodynamic performance of flapping wings (Zheng et al, 2013), is induced by the inertial forces of the flapping wings. The aerodynamic force, however, accounts for the second peak in the deformation that is observed from the middle to the late of each half-stroke (figure 4ai,aii). The deformation is kept at high level until the end of the stroke, possibly because the wing experiences large aerodynamic force by the unsteady mechanisms such as the enlarged leading-edge vortex before breakdown.

We further investigated the effect of the difference in wing deformation on the aerodynamic power consumption and force generation of flapping wings, which points out the great impact of the fluid-structure interaction on the flapping wing aerodynamics. It is shown previously that the increase of the force at the beginning of the half-stroke is due to the delayed burst of the leading-edge vortex (Nakata \& Liu, 2012b). In this study, we further revealed that the delayed burst is caused by the response of the flexible wings to the fluid-structure interaction, rather than the increase in twist or bending due to the inertial force, because such force increase with lower density ratio is quite marginal (figure $6 b$ ). In order to confirm this, the leading-edge vortex and pressure distribution on the surface of rigid and flexible wings with various density ratios at the stroke cycle of 0.16 are visualized in figure 8 . The burst of the leading-edge vortex, and the reduction of the negative pressure, are clearly observed in case of the rigid wing (figure 
$8 a$ ) or the flexible wing at lower density ratio (figure $8 b, c$ ), while the leading-edge vortex is attached on the wing surface when the density ratio is higher (figure $8 d, e_{2} f$ ), which results in higher negative pressure on the surface and hence the higher aerodynamic forces at the timing.

The adjustment of the profile of the translational and rotational velocity at the distal part of the flapping wings further affects the aerodynamic force and power consumption. Maximum aerodynamic powers and forces of flexible wings are increased at lower density ratio, while are reduced down to the level of rigid and flat wings at higher density ratio (figure $6 a$ ), possibly because of the adjustment of the maximum wingtip velocity (figure 5 bii). With higher density ratio, the aerodynamic powers and forces are, however, reduced slowly. This is probably due to the sharp increase of the rotational velocity at the stroke reversal which can greatly enhance the forces due to the wing rotation such as rotational drag (Bomphrey et al, 2017).

The fluid-structure interaction alters not only the profiles, but also the averages of the aerodynamic powers and forces (figure 7). As suggested in a previous study, the aerodynamic forces are increased by the wing deformation regardless of the density ratio (Yin \& Luo, 2010), but the mechanism can be switched from the increase of the translational and rotational velocity at lower density ratio to the delayed burst of the leading-edge vortex at higher density ratio as described above. In spite of the increase in the aerodynamic powers, the efficiency is also enhanced at
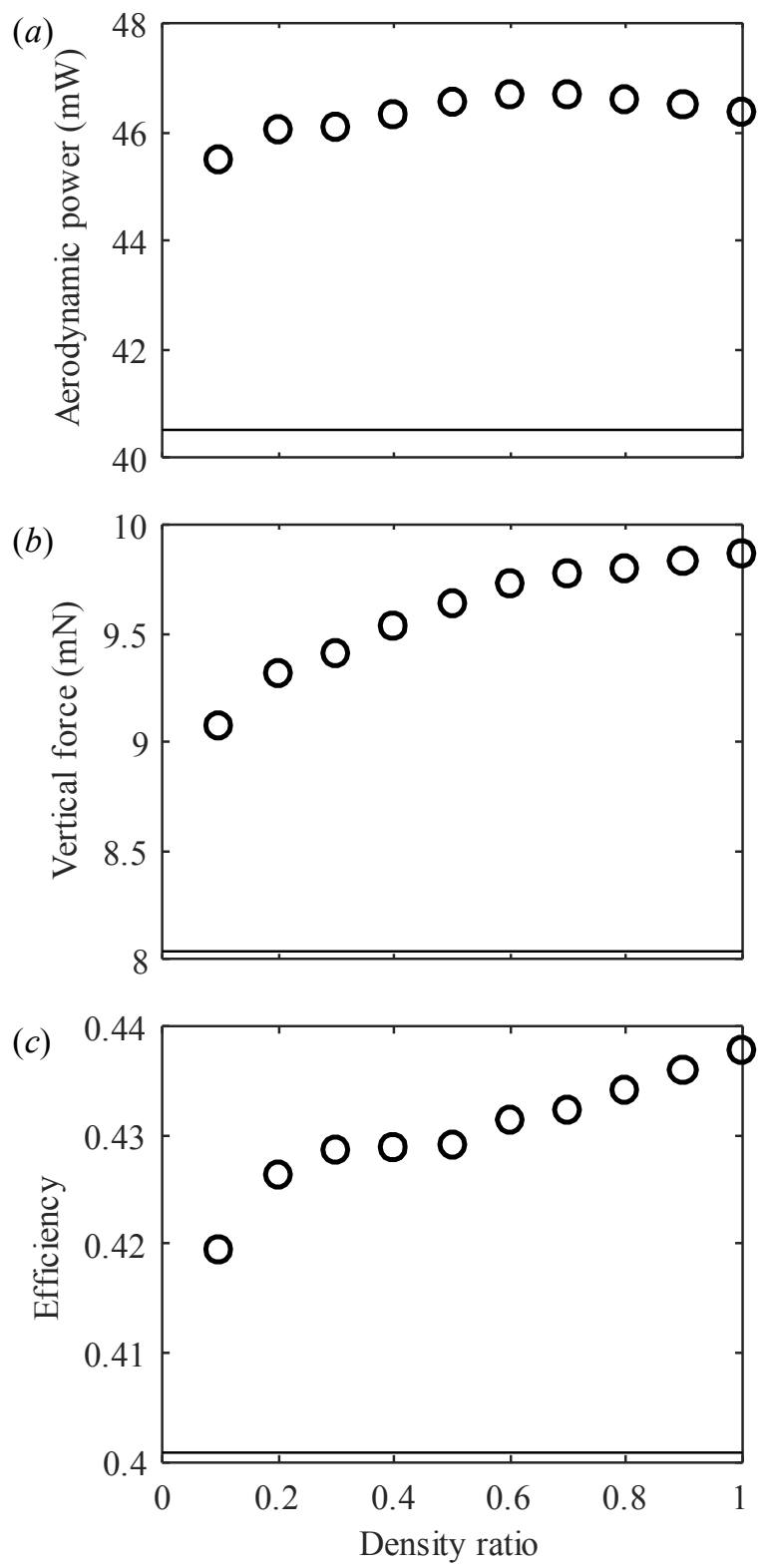

Figure 7 Effect of the density ratio on the cycle-averaged $(a)$ aerodynamic power, $(b)$ vertical force and $(c)$ the aerodynamic efficiency of the single wing. 


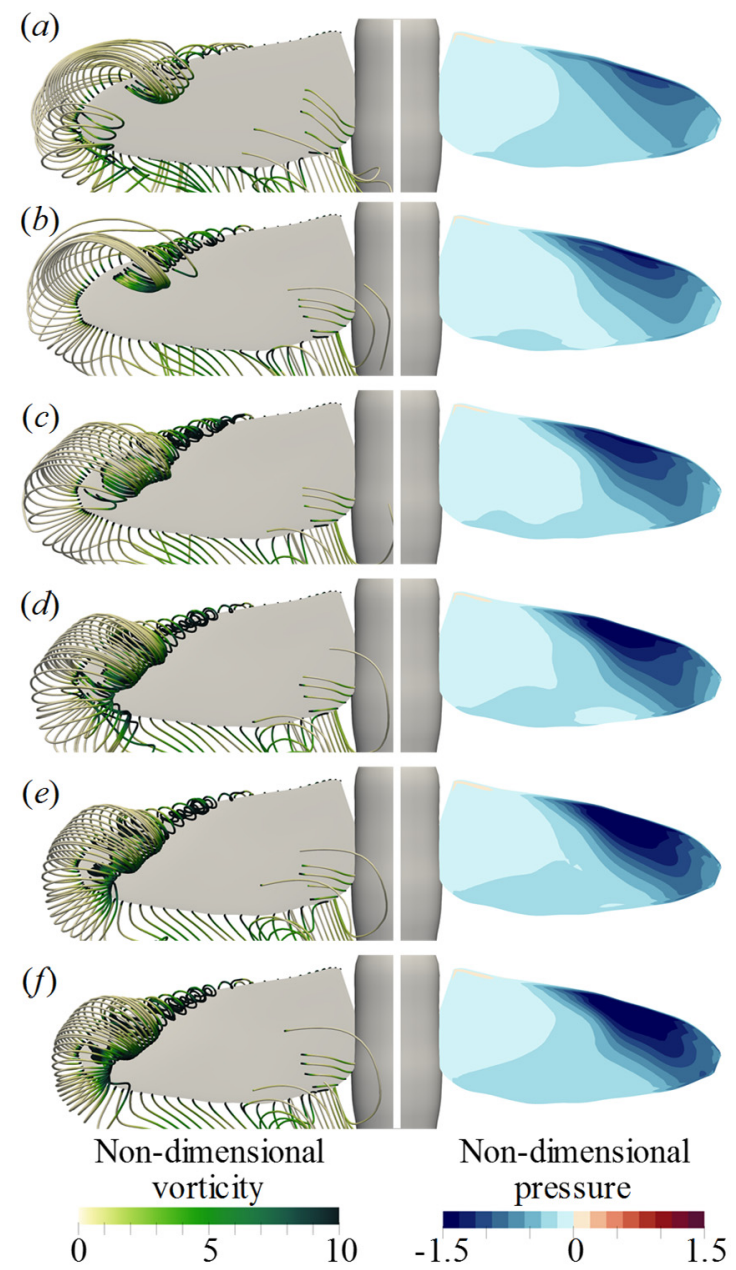

Figure 8 Instantaneous streamlines and pressure distribution on $(a)$ the rigid and the flexble wings with the density ratios of $(b) 0.2,(c) 0.4,(d) 0.6,(e) 0.8$ and $(f) 1.0$.

higher density ratios, possibly because the nose-down twist at the mid-stroke adjust the angle of attack at distal part of the wings favorably (figure $4 a$ ). The efficiency is higher at higher density ratio, because, with the existence of the aerodynamic forces, the twist is further increased so that the wing shape is thought to approach to the optimum shape of flapping wings. The results demonstrated that, while the deformation of the flapping wings, especially the twist, is mainly caused by the inertial forces, the passive adjustment of the wing shape and kinematics through the fluid-structure interaction has a great impact on the aerodynamic performances. The fluid-structure interaction is, therefore, of great importance when designing the flexible flapping wings for the insect-scale micro aerial vehicles.

\section{Conclusion}

In this study, we performed computational fluid-structure interaction analyses to investigate the effect of the aerodynamic force on the deformation and the aerodynamic performance of flexible flapping wings. The effect of the aerodynamic force is adjusted by virtually reducing the air density in the structural analyses of the flexible flapping wings. In terms of the amplitude of the wing deformation, the large part of the wing twist turns out to be induced by the inertial force. By using the deformation with different density ratios, we further showed that the aerodynamic power, force and efficiency can be enhanced by the wing deformation regardless of the density ratio, but, with the existence of the aerodynamic force, the aerodynamic efficiency can be further enhanced by the adjustment of the wing kinematics and the adaptive response of the flexible wings to the unsteady aerodynamics. These results point out the great influence of the fluid-structure interaction on the aerodynamic performance of flapping wings and its importance when designing the flexible wings for the bio-inspired micro aerial vehicles. 


\section{Acknowledgement}

This work was partly supported by the Grant-in-Aid for Scientific Research on Innovative Areas of No. 24120007 , JSPS, and JSPS KAKENHI Grant Number JP17K17638.

\section{References}

Bomphrey, R. J., Nakata, T., Phillips, N. and Walker, S. M. Smart wing rotation and trailing-edge vortices enable high frequency mosquito flight. Nature, Vol.544, No.7648, (2017), pp.92-95.

Combes, S. A. and Daniel, T. L. Into thin air: contributions of aerodynamic and inertial-elastic forces to wing bending in the hawkmoth Manduca sexta. Journal of Experimental Biology, Vol.206, No.17 (2003), pp.2999-3006.

Dickinson, M. H., Lehmann, F. O. and Sane, S. P. Wing rotation and the aerodynamic basis of insect flight. Science, Vol.284, No.5422 (1999), pp.1954-1960.

Ellington, C. P., van den Berg, C., Willmott, A. P. \& Thomas, A. L. R. Leading-edge vortices in insect flight. Nature, Vol.384, No.(1996), pp.626-630.

Le, T. Q., Truong, T. V., Park, S. H., Truong, T. Q., Ko, J. H., Park, H. C. \& Byun, D. Improvement of the aerodynamic performance by wing flexibility and elytra -hind wing interaction of a beetle during forward flight. Journal of the Royal Society Interface, Vol.10, No.85 (2013), 20130312.

Liu, H. Integrated modeling of insect flight: from morphology, kinematics to aerodynamics. Journal of Computational Physics, Vol.228, No.2 (2009), pp.439-459.

Liu, H., Ellington, C. P., Kawachi, K., van den Berg, C. \& Willmott, A. P. A computational fluid dynamic study of hawkmoth hovering. Journal of Experimental Biology, Vol.201, No.4 (1998), pp.461-477.

Liu, H., Ravi, S., Kolomenskiy, D. \& Tanaka, H. Biomechanics and biomimetics in insect-inspired flight systems. Philosophical Transactions of the Royal Society B, Vol.371, No.1704 (2016), 20150390.

Nakata, T. \& Liu, H. A fluid-structure interaction model of insect flight with flexible wings. Journal of Computational Physics, Vol.231, No.4 (2012a), pp.1822-1847.

Nakata, T. \& Liu, H. Aerodynamic performance of a hovering hawkmoth with flexible wings: a computational approach. Proceedings of the Royal Society B, Vol.279, No.1729 (2012b), pp.722-731.

Piperno, S. \& Farhat, C. Partitioned procedures for the transient solution of coupled aeroelastic problems - part II: energy transfer analysis and three-dimensional applications. Computer Methods in Applied Mechanics and Engineering, Vol.190, No.24-25 (2001), pp.3147-3170.

Sridhar, M. \& Kang, C.-K. Aerodynamic performance of two-dimensional, chordwise flexible flapping wings at fruit fly scale in hover flight. Bioinspiration \& Biomimetics, Vol.10, No.3 (2015), 036007.

Srygley, R. B. \& Thomas, A. L. R. Unconventional lift-generating mechanisms in free-flying butterflies. Nature, Vol.420, No.6916 (2002), pp.660-664.

Walker, S. M., Thomas, A. L. R. \& Taylor, G. K. Deformable wing kinematics in the desert locust: how and why do camber, twist and topography vary through the stroke? Journal of the Royal Soceity Interface, Vol.6, No.38 (2009), pp.735-747.

Weis-Fogh, T. Quick estimates of flight fitness in hovering animals, including novel mechanisms for lift production. Journal of Experimental Biology, Vol.59 (1973), pp.169-230.

Willmott, A. P. \& Ellington, C. P. The mechanics of flight in the hawkmoth Manduca sexta. I. kinematics of hovering and forward flight. Journal of Experimental Biology, Vol.200 (1997), pp.2705-2722.

Wootton, R. J. Support and deformability in insect wings. J. Zool., Vol.193, No.4 (1981), pp.447-468.

Wootton, R. J. Functional Morphology of Insect Wings. Annual Review of Entomology, Vol.37 (1992), pp.113-140.

Yin, B. \& Luo, H. Effect of wing inertia on hovering performance of flexible flapping wings. Physics of Fluids, Vol.22, No.11 (2010), 111902.

Young, J., Walker, S. M., Bomphrey, R. J., Taylor, G. K. \& Thomas, A. L. R. Details of insect wing design and deformation enhance aerodynamic function and flight efficiency. Science, Vol.325, No.5947 (2009), pp.1549-1552.

Zheng, L., Hedrick, T. L. \& Mittal, R. Time-Varying Wing-Twist Improves Aerodynamic Efficiency of Forward Flight in Butterflies. PLoS ONE, Vol.8, No.1 (2013), e53060. 